\title{
Closed formula for the metric in the Hilbert space of a $\mathcal{P} \mathcal{T}$-symmetric model
}

\author{
D. KREJČIŘÍK ${ }^{1}$, H. BÍlA ${ }^{1,2}$ AND M. ZNOJIL ${ }^{1}$ \\ 1 Department of Theoretical Physics, Nuclear Physics Institute, \\ Academy of Sciences, 25068 Rež near Prague, Czech Republic \\ 2 Faculty of Mathematics and Physics, Charles University in Prague, \\ Ke Karlovu 3, 12116 Praha 2
}

24 April 2006

\begin{abstract}
We introduce a very simple, exactly solvable $\mathcal{P} \mathcal{T}$-symmetric non-Hermitian model with real spectrum, and derive a closed formula for the metric operator which relates the problem to a Hermitian one.
\end{abstract}

\section{Introduction}

In a way motivated by the needs of nuclear physics, Scholtz, Geyer and Hahne 1] established a general mathematical framework for the consistent formulation of quantum mechanics where a set of observables are represented by bounded nonHermitian operators $A_{1}, \ldots, A_{N}$ with real spectra in a Hilbert space $\mathcal{H}$. In essence, they conjectured that in the similar situations one may find a bounded positive Hermitian operator $\Theta$, called metric, which fulfils

$$
A_{k}^{*} \Theta=\Theta A_{k} \quad \text { for all } \quad k \in\{1, \ldots, N\},
$$

where $A_{k}^{*}$ denotes the adjoint operator of $A_{k}$ in $\mathcal{H}$.

Several years later, the notion of the metric operator $\Theta$ re-emerged as a particularly useful mathematical tool in the context of the so-called $\mathcal{P} \mathcal{T}$-symmetric quantum mechanics 2, 3. In this framework people usually paid attention to the systems with a single observable, viz, with a Hamiltonian $A_{1} \equiv H \neq H^{*}$ which possesses real spectrum and for which the Schrödinger equation is invariant under a simultaneous change of spatial reflection $\mathcal{P}$ and time reversal $\mathcal{T}$.

In the current literature a lot of effort has been devoted to the study of the particular models of $H$. For their more detailed reviews and discussion the reader is referred to the proceedings of the International Workshops on Pseudo-Hermitian Hamiltonians in Quantum Physics 4, 5, 6]. One finds that the construction of a non-trivial operator $\Theta \neq I$, however difficult, is a key to the 
correct probabilistic interpretation of all the $\mathcal{P} \mathcal{T}$-symmetric quantum systems [7, 8, 9, 10]. Indeed, it defines "the physical" inner product $(\cdot, \cdot)_{\Theta}:=(\cdot, \Theta \cdot)$ which makes the Hamiltonian $H$ "Hermitian" or, in the language of [1], quasiHermitian. For this reason, there have been many attempts to calculate the metric operator $\Theta$ for the various $\mathcal{P} \mathcal{T}$-symmetric models of interest 11, 12, 13, 14, 15, 16, 17, 18, 19. Because of the complexity of the problem, however, it is not surprising that most of the available formulae for $\Theta$ are just approximative, usually expressed as leading terms of perturbation series [17.

The authors of [1] discussed why our knowledge of the new inner product was necessary for the evaluation of the physical predictions. They emphasized that the theory endowed with it is a genuine quantum theory satisfying all the necessary postulates. In a fairly recent continuation of this discussion 20] it has been underlined that in the infinite-dimensional Hilbert spaces $\mathcal{H}$ the requirement of the boundedness of the metric operator $\Theta$ plays a key role and that it deserves an extremely careful analysis in the applications where a naïve approach may lead to wrong results. In some sense, our present paper may be read as a direct continuation of the rigorous mathematical discussion in [20].

In particular we are going to illustrate here that our understanding of (1) for unbounded operators $H$ as the identity on functions from the operator domain of $H$ (cf (9) below) requires that $\Theta$ maps the operator domain of $H$ into the operator domain of the adjoint $H^{*}$. In such a setting we imagined that the best way of finding a support for such an argument can be sought in some exactly solvable $\mathcal{P} \mathcal{T}$-symmetric model. We decided to develop a new one - such that its metric can be obtained in a closed formula and in a rigorous manner.

The model we deal with in the present paper is one-dimensional, defined in the Hilbert space

$$
\mathcal{H}:=L^{2}((0, d))
$$

where $d$ is a given positive number. In this Hilbert space we consider the Hamiltonian $H_{\alpha}$ which acts as the Laplacian, i.e.,

$$
H_{\alpha} \psi:=-\psi^{\prime \prime},
$$

and satisfies the following Robin boundary conditions:

$$
\psi^{\prime}(0)+i \alpha \psi(0)=0 \quad \text { and } \quad \psi^{\prime}(d)+i \alpha \psi(d)=0 .
$$

Here $\psi$ is a function from the Sobolev space $W^{2,2}((0, d))$ and $\alpha$ is a real constant. That is, the operator domain $D\left(H_{\alpha}\right)$ consists of functions with integrable (generalized) derivatives up to the second order and satisfying (2) at the boundary points. Because of the nature of the boundary conditions, $H_{\alpha}$ is not Hermitian unless $\alpha=0$, but it is $\mathcal{P} \mathcal{T}$-symmetric with the operators $\mathcal{P}$ and $\mathcal{T}$ being defined by $(\mathcal{P} \psi)(x):=\psi(d-x)$ and $\mathcal{T} \psi:=\bar{\psi}$, respectively.

It seems that our Hamiltonian $H_{\alpha}$ represents the simplest $\mathcal{P} \mathcal{T}$-symmetric model whatsoever. The fact that the support of the non-Hermitian perturbation is of measure zero invokes the $\mathcal{P} \mathcal{T}$-symmetric models [21, 22, 23] involving complex point interactions. But our model is even simpler, since it does not 
require any matching of solutions known explicitly off the points where the $\delta$-interaction is supported.

Indeed, the non-Hermiticity of $H_{\alpha}$ enters through the boundary conditions only, while the Hamiltonian models a free quantum particle in the interval $(0, d)$. Consequently, the spectral problem for $H_{\alpha}$ can be solved explicitly in terms of sines and cosines ( $c f$ Section 3 for more details). Furthermore, an explicit form for the eigenfunctions enables us to obtain a remarkably simple expression for the metric operator:

Theorem 1. Let $\Theta(\alpha)$ be the linear operator defined in $\mathcal{H}$ by

$$
\Theta(\alpha):=I+\phi_{0}^{\alpha}\left(\phi_{0}^{\alpha}, \cdot\right)+\Theta_{0}+i \alpha \Theta_{1}+\alpha^{2} \Theta_{2},
$$

where I denotes the identity operator in $\mathcal{H},(\cdot, \cdot)$ is the inner product on $\mathcal{H}$, antilinear in the first factor and linear in the second one,

$$
\phi_{0}^{\alpha}(x):=\sqrt{\frac{1}{d}} \exp (i \alpha x)
$$

and the operators $\Theta_{0}, \Theta_{1}$ and $\Theta_{2}$ acts in $\mathcal{H}$ as

$$
\begin{aligned}
& \left(\Theta_{0} \psi\right)(x):=-\frac{1}{d}(J \psi)(d), \\
& \left(\Theta_{1} \psi\right)(x):=2(J \psi)(x)-\frac{x}{d}(J \psi)(d)-\frac{1}{d}\left(J^{2} \psi\right)(d), \\
& \left(\Theta_{2} \psi\right)(x):=-\left(J^{2} \psi\right)(x)+\frac{x}{d}\left(J^{2} \psi\right)(d),
\end{aligned}
$$

with

$$
(J \psi)(x):=\int_{0}^{x} \psi .
$$

Then $\Theta(\alpha)$ is bounded, symmetric, non-negative and satisfies

$$
\forall \psi \in D\left(H_{\alpha}\right), \quad H_{\alpha}^{*} \Theta(\alpha) \psi=\Theta(\alpha) H_{\alpha} \psi .
$$

Furthermore, $\Theta(\alpha)$ is positive if the condition

$$
\alpha d / \pi \notin \mathbb{Z} \backslash\{0\}
$$

holds true.

Note that the metric $\Theta(\alpha)$ tends to the identity operator $I$ as $\alpha \rightarrow 0$, which is expected due to the fact that $H_{0}$ is nothing else than the (self-adjoint) Neumann Laplacian in $\mathcal{H}$. The condition (10) ensures that all the eigenvalues of $H_{\alpha}$ are simple. For simplicity, we do not consider the degenerate cases in the present paper.

This paper is organized as follows. In the following Section 2 we introduce the Hamiltonian $H_{\alpha}$ by means of its associated quadratic form; this provides an elegant way of showing that the operator is closed. The spectral problem 
for $H_{\alpha}$ is considered in Section [3] in particular, we show that the spectrum is real and discrete, and write down the explicit eigenfunctions and eigenvalues. Section 4 contains the main idea of the present paper; namely, we observe that the eigenfunctions of $H_{\alpha}$ are expressed in terms of Dirichlet and Neumann complete orthonormal families in the interval $(0, d)$ and use a special normalization to simplify the eigenfunctions of the adjoint $H_{\alpha}^{*}$. These enable us, in Section 5 . to evaluate certain infinite series defining the metric operator and prove Theorem 1. We conclude the paper by Section [6] where we add several remarks and discuss a possible extension of our model.

\section{The Hamiltonian}

Let us first introduce the operator $H_{\alpha}$ in a proper way. We start with the associated sesquilinear form $h_{\alpha}$ defined in the Hilbert space $\mathcal{H}$ by the domain $D\left(h_{\alpha}\right):=W^{1,2}((0, d))$ and by the prescription:

$$
h_{\alpha}(\phi, \psi):=\left(\phi^{\prime}, \psi^{\prime}\right)+i \alpha \overline{\phi(d)} \psi(d)-i \alpha \overline{\phi(0)} \psi(0) \text {. }
$$

Here $(\cdot, \cdot)$ denotes the standard inner product on $\mathcal{H}$; the corresponding norm will be denoted by $\|\cdot\|$.

Note that the boundary terms in (11) are well defined because the domain of the quadratic form is embedded in the space of uniformly continuous functions on $(0, d)$ due to the Sobolev embedding theorem [24]. It is also known that the Sobolev space $W^{1,2}((0, d))$ is dense in $\mathcal{H}$; hence $h_{\alpha}$ is densely defined. Moreover, the real part of $h_{\alpha}$, denoted by $\Re h_{\alpha}$, is a densely defined, symmetric, positive, closed sesquilinear form (since it corresponds to the self-adjoint Neumann Laplacian in $\mathcal{H}$ ). Of course, $h_{\alpha}$ itself is not symmetric unless $\alpha=0$, however, it can be shown that it is sectorial and closed. To see it, we use [25, Thm. VI.1.33] and prove that the imaginary part of $h_{\alpha}$, denoted by $\Im h_{\alpha}$, is a small perturbation of $\Re h_{\alpha}$ in the following sense:

Lemma 1. $\Im h_{\alpha}$ is relatively bounded with respect to $\Re h_{\alpha}$, with

$$
\left|\left(\Im h_{\alpha}\right)[\psi]\right| \leq \epsilon^{-1} \alpha^{2}\|\psi\|^{2}+\epsilon\left(\Re h_{\alpha}\right)[\psi]
$$

for all $\psi \in W^{1,2}((0, d))$ and any positive constant $\epsilon$.

Proof. Writing $|\psi(d)|^{2}-|\psi(0)|^{2}=\int_{0}^{d}\left(|\psi|^{2}\right)^{\prime}=2 \Re\left(\psi, \psi^{\prime}\right)$, and applying the Schwarz and Cauchy inequalities to the last term, we obtain the desired result.

In view of the above properties and the first representation theorem 25 , Thm. VI.2.1], there exists a unique $m$-sectorial operator $H_{\alpha}$ in $\mathcal{H}$ such that $h_{\alpha}(\phi, \psi)=\left(\phi, H_{\alpha} \psi\right)$ for all $\phi \in D\left(h_{\alpha}\right)$ and $\psi \in D\left(H_{\alpha}\right) \subset D\left(h_{\alpha}\right)$. The operator domain $D\left(H_{\alpha}\right)$ consists of those functions $\psi \in D\left(h_{\alpha}\right)$ for which there exists 
$\eta \in \mathcal{H}$ such that $h_{\alpha}(\phi, \psi)=(\phi, \eta)$ holds for every $\phi \in D\left(h_{\alpha}\right)$. Furthermore, using the ideas of [25. Ex. VI.2.16], it is possible to verify that indeed

$$
\begin{aligned}
H_{\alpha} \psi & =-\psi^{\prime \prime} \\
\psi \in D\left(H_{\alpha}\right) & =\left\{\psi \in W^{2,2}((0, d)) \mid \psi \text { satisfies (2) }\right\} .
\end{aligned}
$$

The above procedure also implies that the adjoint operator $H_{\alpha}^{*}$ is simply obtained by the replacement $\alpha \mapsto-\alpha$.

Summing up the results, we obtain:

Proposition 1. The operator $H_{\alpha}$ defined by [12) is $m$-sectorial in $\mathcal{H}$ and satisfies

$$
H_{\alpha}^{*}=H_{-\alpha} .
$$

\section{The spectrum}

An important property of an operator being $m$-sectorial is that it is closed. Then, in particular, the spectrum is well defined by means of the resolvent operator. We claim that our $H_{\alpha}$ is an operator with compact resolvent. This can be seen by noticing that the Neumann Laplacian $H_{0}$ (associated with $\Re h_{\alpha}$ ) is an operator with compact resolvent and by using the perturbation result of [25. Thm. VI.3.4] together with Lemma 11 Consequently, we know that the spectrum of $H_{\alpha}$, denoted by $\sigma\left(H_{\alpha}\right)$, is purely discrete, i.e., it consists entirely of isolated eigenvalues with finite (algebraic) multiplicities.

The eigenvalue problem $H_{\alpha} \psi=k^{2} \psi$, with $k \in \mathbb{C}$, can be solved explicitly in terms of sines and cosines. In particular, the boundary conditions lead to the following implicit equation for the eigenvalues:

$$
\left(k^{2}-\alpha^{2}\right) \sin (k d)=0 .
$$

That is,

$$
\sigma\left(H_{\alpha}\right)=\left\{\alpha^{2}\right\} \cup\left\{k_{j}^{2}\right\}_{j=1}^{\infty}, \quad \text { where } \quad k_{j}:=j \pi / d .
$$

Hereafter we shall use the index $j \in \mathbb{N}$ to count the eigenvalues as in (14), with the convention that the eigenvalue for $j=0$ is given by $\alpha^{2}$.

While the spectrum of $H_{\alpha}$ is real, it exhibits important differences with respect to the spectra of self-adjoint one-dimensional differential operators. For instance, the spectrum of $H_{\alpha}$ may not be simple and even the lowest eigenvalue may be degenerate for particular choices of $\alpha$. Notice also that $H_{\alpha}$ coincides with the spectrum of the Neumann Laplacian $H_{0}$ up to the lowest (zero) eigenvalue which is shifted to $\alpha^{2}$.

In this paper we restrict to the non-degenerate case, i.e., we make the hypothesis (10). Then the eigenfunctions of $H_{\alpha}$ corresponding to (14) with the convention mentioned there are given by

$$
\psi_{j}^{\alpha}(x):= \begin{cases}A_{0}^{\alpha} \exp (-i \alpha x) & \text { if } \quad j=0 \\ A_{j}^{\alpha}\left(\cos \left(k_{j} x\right)-i \frac{\alpha}{k_{j}} \sin \left(k_{j} x\right)\right) & \text { if } \quad j \geq 1\end{cases}
$$


where each $A_{j}$ is an arbitrary non-zero complex number. In view of Proposition 11 the spectrum of the adjoint $H_{\alpha}^{*}$ coincides with (14) and the corresponding eigenfunctions are given by

$$
\phi_{j}^{\alpha}(x):= \begin{cases}B_{0}^{\alpha} \exp (i \alpha x) & \text { if } \quad j=0, \\ B_{j}^{\alpha}\left(\cos \left(k_{j} x\right)+i \frac{\alpha}{k_{j}} \sin \left(k_{j} x\right)\right) & \text { if } \quad j \geq 1,\end{cases}
$$

where each $B_{j}$ is again an arbitrary non-zero complex number.

We collect the obtained spectral results into the following proposition:

Proposition 2. The spectrum of $H_{\alpha}$ is real and consists of discrete eigenvalues specified in (14). If the condition (10) holds, then all the eigenvalues have multiplicity one and the corresponding eigenfunctions are given by (15).

\section{Special normalization}

It follows directly by combining the eigenvalue problems for $H_{\alpha}$ and its adjoint that $\phi_{j}^{\alpha}$ and $\psi_{k}^{\alpha}$ are orthogonal to each other provided $j \neq k$ and the nondegeneracy condition (10) holds. The stronger result

$$
\forall j, k \in \mathbb{N}, \quad\left(\phi_{j}^{\alpha}, \psi_{k}^{\alpha}\right)=\delta_{j k}
$$

will hold provided we normalize the eigenfunctions in a special way. Namely, (17) follows by choosing the coefficients $A_{j}^{\alpha}$ and $B_{j}^{\alpha}$ according to the equations

$$
\begin{aligned}
& 1=A_{0}^{\alpha} \overline{B_{0}^{\alpha}} \frac{1-\exp (-2 i \alpha d)}{2 i \alpha}, \\
& 1=A_{j}^{\alpha} \overline{B_{j}^{\alpha}} \frac{\left(k_{j}^{2}-\alpha^{2}\right) d}{2 k_{j}^{2}} \quad \text { for } \quad j \geq 1 .
\end{aligned}
$$

(If $\alpha=0$, the fraction in the first equation should be understood as the expression obtained after taking the limit $\alpha \rightarrow 0$.) These equations can clearly be satisfied as soon as (10) holds.

We still have a freedom in specifying $A_{j}^{\alpha}$ and $B_{j}^{\alpha}$. For further purposes, however, we choose the coefficients $B_{j}^{\alpha}$ in a very simple form by the requirements

$$
B_{0}:=\sqrt{1 / d} \quad \text { and } \quad B_{j}:=\sqrt{2 / d} \quad \text { for } \quad j \geq 1,
$$

while we leave more complicated formula, determined by the equations (18) and (19), for the coefficients $A_{j}^{\alpha}$. Then $\phi_{0}^{\alpha}$ coincides with (4) and we have the decomposition

$$
\phi_{j}^{\alpha}(x)=\chi_{j}^{N}(x)+i \frac{\alpha}{k_{j}} \chi_{j}^{D}(x) \quad \text { for } \quad j \geq 1,
$$

where $\left\{\chi_{j}^{N}\right\}_{j=0}^{\infty}$, respectively $\left\{\chi_{j}^{D}\right\}_{j=1}^{\infty}$, denotes the set of normalized eigenfunctions of the Neumann, respectively Dirichlet, Laplacian in $\mathcal{H}$ :

$$
\chi_{j}^{N}(x):=\left\{\begin{array}{ll}
\sqrt{1 / d} & \text { if } \quad j=0, \\
\sqrt{2 / d} \cos \left(k_{j} x\right) & \text { if } \quad j \geq 1,
\end{array} \quad \chi_{j}^{D}(x):=\sqrt{2 / d} \sin \left(k_{j} x\right) .\right.
$$


In addition to (21), we also have the uniform convergence $\phi_{0}^{\alpha} \rightarrow \chi_{0}^{N}$ as $\alpha \rightarrow 0$. We point out the result we shall need later:

Proposition 3. If the condition (10) holds true, then the eigenfunctions $\psi_{j}^{\alpha}$ of $H_{\alpha}$ and the eigenfunctions $\phi_{j}^{\alpha}$ of $H_{\alpha}^{*}$ can be chosen in such a way that they satisfy the biorthonormality relations (17) and the latter satisfy (21).

The decomposition (21) plays a crucial role in the subsequent section, mainly due to the fact that $\left\{\chi_{j}^{N}\right\}_{j=0}^{\infty}$ and $\left\{\chi_{j}^{D}\right\}_{j=1}^{\infty}$ are well known to form complete orthonormal families. In particular, we have the expansions

$$
\psi=\sum_{j=0}^{\infty} \chi_{j}^{N}\left(\chi_{j}^{N}, \psi\right)=\sum_{j=1}^{\infty} \chi_{j}^{D}\left(\chi_{j}^{D}, \psi\right)
$$

and the Parseval equalities

$$
\|\psi\|^{2}=\sum_{j=0}^{\infty}\left|\left(\chi_{j}^{N}, \psi\right)\right|^{2}=\sum_{j=1}^{\infty}\left|\left(\chi_{j}^{D}, \psi\right)\right|^{2}
$$

for every $\psi \in \mathcal{H}$.

\section{The metric}

With an abuse of notation, we initially define

$$
\Theta(\alpha):=\sum_{j=0}^{\infty} \phi_{j}^{\alpha}\left(\phi_{j}^{\alpha}, \cdot\right)
$$

and show that this operator can be cast into the form (3) with (4)-(7). In fact, using (21) and (22), it is readily seen that (3) holds with

$$
\Theta_{0}:=-\chi_{0}^{N}\left(\chi_{0}^{N}, \cdot\right)
$$

and

$$
\Theta_{1}:=\sum_{j=1}^{\infty} \frac{\chi_{j}^{D}\left(\chi_{j}^{N}, \cdot\right)-\chi_{j}^{N}\left(\chi_{j}^{D}, \cdot\right)}{k_{j}}, \quad \Theta_{2}:=\sum_{j=1}^{\infty} \frac{\chi_{j}^{D}\left(\chi_{j}^{D}, \cdot\right)}{k_{j}^{2}}
$$

Recalling the definition (8) of the bounded integral operator $J$ in $\mathcal{H}$, it is evident that the rank-one operator (25) can be expressed in terms of $J$ as in (5). It remains to verify that (26) can be expressed as in (6) and (7).

First of all, we notice that the operator (24) is well defined in the sense that $\Theta_{1}$ and $\Theta_{2}$ as defined in (26) are bounded linear operators in $\mathcal{H}$. This can be seen by using (23) and the Schwarz inequality. Actually, the series in (26) are uniformly convergent, and $\Theta_{2}$ can be written as an integral Hilbert-Schmidt operator, but we will not use these facts. Our way to sum up the infinite series is based on the following lemma: 


\section{Lemma 2.}

$$
\sum_{j=1}^{\infty} \frac{\chi_{j}^{D}(x) \chi_{j}^{N}(d)}{k_{j}}=-\frac{x}{d} \quad \text { uniformly for all } \quad x \in[0, d] .
$$

Proof. The series is uniformly convergent due to Abel's uniform convergence test. Let $l$ denote the identity function on $(0, d)$, i.e. $l(x):=x$. Using the expansion (22) and integrating by parts, we get

$$
l=\sum_{j=1}^{\infty} \chi_{j}^{D}\left(\chi_{j}^{D}, l\right)=\sum_{j=1}^{\infty} \chi_{j}^{D}\left(\left(-\chi_{j}^{N} / k_{j}\right)^{\prime}, l\right)=-\sum_{j=1}^{\infty} \chi_{j}^{D} \chi_{j}^{N}(d) d / k_{j},
$$

where the last equality follows from the fact that all $\chi_{j}^{N}$ with $j \geq 1$ are orthogonal to the constant function $\chi_{0}^{N}$. This concludes the proof.

Since $J \psi$ is an indefinite integral of $\psi$ and $(J \psi)(0)=0$, an integration by parts yields for every $\psi \in \mathcal{H}$ :

$$
\begin{aligned}
& \left(\chi_{j}^{N}, \psi\right)=k_{j}\left(\chi_{j}^{D}, J \psi\right)+\chi_{j}^{N}(d)(J \psi)(d), \\
& \left(\chi_{j}^{D}, \psi\right)=-k_{j}\left(\chi_{j}^{N}, J \psi\right)=-k_{j}^{2}\left(\chi_{j}^{D}, J^{2} \psi\right)-k_{j} \chi_{j}^{N}(d)\left(J^{2} \psi\right)(d) .
\end{aligned}
$$

Incorporating these identities into (26) and using (22) together with Lemma 2 we obtain the formulae (6) and (7) for (26).

Now we are in a position to prove Theorem 1

Proof of Theorem [1. The boundedness of (3) is clear; in particular, crude estimates yield

$$
\|\Theta(\alpha) \psi\| \leq\left(3+4 \alpha d+2 \alpha^{2} d^{2}\right)\|\psi\|
$$

for every $\psi \in \mathcal{H}$.

Integrating by parts, it is also easy to check that the identity

$$
(\psi, \Theta(\alpha) \psi)=\left|\left(\phi_{0}^{\alpha}, \psi\right)\right|^{2}+\|\psi+i \alpha J \psi\|^{2}-\left|\left(\chi_{0}^{N}, \psi+i \alpha J \psi\right)\right|^{2}
$$

holds for every $\psi \in \mathcal{H}$, where the right hand side is real-valued and non-negative due to (23). This proves that $\Theta(\alpha)$ is symmetric and non-negative.

Let us show that $\Theta(\alpha)$ is positive provided (10) holds. If the right hand side of (27) were equal to zero with a non-zero $\psi \in \mathcal{H}$, then the first Parseval equality in (23) would imply that the function $\psi+i \alpha J \psi$ must be constant, being orthogonal to all functions orthogonal to 1 . Consequently, $\psi$ is proportional to $\psi_{0}^{\alpha}$ and an explicit calculation yields

$$
\left|\left(\phi_{0}^{\alpha}, \psi\right)\right|=\left|\frac{\sin (\alpha d)}{\alpha d}\right|\|\psi\|,
$$

which is clearly positive for all $\alpha$ satisfying (10). 
Finally, let us comment on the identity (9). Let $\psi \in D\left(H_{\alpha}\right)$. We first note that it straightforward to check that $\Theta(\alpha) \psi$ belongs to $D\left(H_{\alpha}^{*}\right)$, so that the left hand side of (9) makes sense. We also have

$$
-(\Theta(\alpha) \psi)^{\prime \prime}=-\psi^{\prime \prime}-2 i \alpha \psi^{\prime}+\alpha^{2} \psi+\alpha^{2} \phi_{0}^{\alpha}\left(\phi_{0}^{\alpha}, \psi\right)=-\Theta(\alpha) \psi^{\prime \prime} .
$$

Here the first equality follows at once, while the second one is not trivial, but it can be verified by using a number of integrations by parts.

This concludes the proof of Theorem [

\section{Concluding remarks}

\subsection{Alternative proofs of the reality of the spectrum}

Recall that $\mathcal{P} \mathcal{T}$-symmetry itself is not sufficient to guarantee the reality of the spectrum of a non-Hermitian operator (see, e.g., [26 27]). Moreover, the existing proofs of the reality [28, 29, 30, 31] are based on rather advanced techniques. Therefore we find it interesting that the reality of the eigenvalues of our Hamiltonian $H_{\alpha}$ can be deduced directly from the structure of the operator, without solving the eigenvalue problem explicitly.

To see it, we rewrite the eigenvalue problem $H_{\alpha} \psi=k^{2} \psi$ using the unitary transform $\psi \mapsto \phi_{0}^{\alpha} \psi:=\phi$ into the boundary value problem

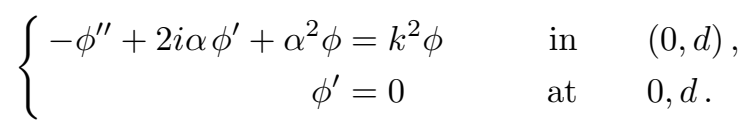

Now we multiply the first equation in (28) by $\overline{\phi^{\prime \prime}}$ and integrate over $(0, d)$. We also multiply the complex conjugation of the first equation in (28) by $\phi^{\prime \prime}$ and integrate over $(0, d)$. Then we subtract the results and use various integrations by parts together with the Neumann boundary conditions to get the identity

$$
\Im\left(k^{2}\right)\left\|\phi^{\prime}\right\|^{2}=0 .
$$

Consequently, either the eigenvalue $k^{2}$ is real or the corresponding eigenfunction $\phi$ is constant. It remains to realize that also the latter implies the former in view of (28).

Finally, let us mention that $H_{\alpha}$ can be reconsidered as a self-adjoint operator in a Krein space [29]. Then the reality of the spectrum of $H_{\alpha}$ for $|\alpha|<\pi / d$ follows from [29] Corol. 3.3]. An alternative proof of the reality of the spectrum of $H_{\alpha}$ for small $\alpha$ also follows from the perturbation result of [30].

\subsection{Biorthonormal basis}

It is easily seen that the operator $\Theta(\alpha)$ defined by (24) formally satisfies (9), with the inverse given by $\Theta(\alpha)^{-1}=\sum_{j=0}^{\infty} \psi_{j}^{\alpha}\left(\psi_{j}^{\alpha}, \cdot\right)$, provided $\left\{\psi_{j}^{\alpha}\right\}_{j=0}^{\infty}$ and $\left\{\phi_{j}^{\alpha}\right\}_{j=0}^{\infty}$ 
fulfil in addition to (17) the following biorthonormal-basis-type relation:

$$
\forall \psi \in \mathcal{H}, \quad \psi=\sum_{j=0}^{\infty} \psi_{j}^{\alpha}\left(\phi_{j}^{\alpha}, \psi\right) .
$$

By "formally" we mean that one has to justify an interchange of summation and differentiation. We did not pursue this direction in the present paper. Instead, we summed up the infinite series (24) using the special normalization (20) leading to (21), and checked that the resulting operator indeed satisfies (1) in the sense of (9).

Nevertheless, let us show that the expansion (29) holds:

Proposition 4. If the condition (10) holds true, then the eigenfunctions $\psi_{j}^{\alpha}$ of $H_{\alpha}$ and the eigenfunctions $\phi_{j}^{\alpha}$ of $H_{\alpha}^{*}$ can be chosen in such a way that (29) is satisfied.

Proof. Assume the special normalization of Section 4 Let us first verify that $\left\{\psi_{j}\right\}_{j=0}^{\alpha}$ is a basis of $\mathcal{H}$, i.e.,

$$
\forall \psi \in \mathcal{H}, \quad \psi=\sum_{j=0}^{\infty} c_{j}^{\psi} \psi_{j}^{\alpha},
$$

where $\left\{c_{j}^{\psi}\right\}_{j=0}^{\infty}$ is a unique sequence of complex numbers. Note that the equality in (30) should be understood as a limit in the norm topology of $\mathcal{H}$; in particular, (30) implies the weak convergence

$$
\forall \phi, \psi \in \mathcal{H}, \quad(\phi, \psi)=\lim _{m \rightarrow \infty}\left(\phi, \sum_{n=1}^{m} c_{j}^{\psi} \psi_{j}^{\alpha}\right) .
$$

Substituting $\psi=0$ and $\phi=\phi_{k}^{\alpha}, k \in \mathbb{N}$, into (31), the biorthonormality relations (17) yield that (30) with $\psi=0$ implies that all $c_{j}^{0}=0$. At the same time,

$$
\left\|\psi_{j}^{\alpha}-\chi_{j}^{N}\right\|^{2}=\alpha^{2} \frac{k_{j}^{2}+\alpha^{2}}{\left(k_{j}^{2}-\alpha^{2}\right)^{2}} \quad \text { for } \quad j \geq 1
$$

and since the right hand side behaves as $\mathcal{O}\left(j^{-2}\right)$ as $j \rightarrow \infty$, we have

$$
\sum_{j=0}^{\infty}\left\|\psi_{j}^{\alpha}-\chi_{j}^{N}\right\|^{2}<\infty .
$$

Consequently, $\left\{\psi_{j}\right\}_{j=0}^{\alpha}$ is a basis of $\mathcal{H}$ due to [25. Thm. V.2.20]. Finally, substituting $\phi=\phi_{k}^{\alpha}, k \in \mathbb{N}$, into (31), the biorthonormality relations (17) yield that $c_{j}^{\psi}=\left(\phi_{j}^{\alpha}, \psi\right)$ for all $j \in \mathbb{N}$.

The same argument also implies the following expansion:

$$
\forall \psi \in \mathcal{H}, \quad \psi=\sum_{j=0}^{\infty} \phi_{j}^{\alpha}\left(\psi_{j}^{\alpha}, \psi\right) .
$$




\subsection{A more general model}

For simplicity, we required that $\alpha$ was real in the present paper. A more general model is given by the following more general $\mathcal{P} \mathcal{T}$-symmetric boundary conditions:

$$
\psi^{\prime}(0)+(\beta+i \alpha) \psi(0)=0 \quad \text { and } \quad-\psi^{\prime}(d)+(\beta-i \alpha) \psi(d)=0,
$$

where $\alpha$ and $\beta$ are real constants. A straightforward modification of the approach of Section 2 ( $c f$ also the first paragraph of Section 3) yields:

Proposition 5. The operator $H_{\alpha, \beta}$ defined in $\mathcal{H}$ by

$$
\begin{aligned}
H_{\alpha, \beta} \psi & =-\psi^{\prime \prime}, \\
\psi \in D\left(H_{\alpha, \beta}\right) & =\left\{\psi \in W^{2,2}((0, d)) \mid \psi \text { satisfies (32) }\right),
\end{aligned}
$$

is an m-sectorial operator with compact resolvent and satisfies $H_{\alpha, \beta}^{*}=H_{-\alpha, \beta}$.

The eigenvalue problem $H_{\alpha, \beta} \psi=k^{2} \psi$, with $k \in \mathbb{C}$, can again be solved in terms of sines and cosines, and one gets the following implicit equation for the eigenvalues:

$$
\left[k^{2}-\left(\alpha^{2}+\beta^{2}\right)\right] \sin (k d)-2 \beta k \cos (k d)=0 .
$$

The main difference with respect to the case $\beta=0$ studied in the present paper is that $H_{\alpha, \beta}$ can possess non-real complex conjugate eigenvalues for $\beta \neq 0$.

\section{Acknowledgment}

One of the authors (D.K.) would like to thank the foundation Český literární fond for a financial support which enabled him to participate in the 3rd International Workshop on Pseudo-Hermitian Hamiltonians in Quantum Physics (Istanbul, 2005). The work has partially been supported by the Czech Academy of Sciences and its Grant Agency within the projects IRP AV0Z10480505 and A100480501

\section{References}

[1] Scholtz FG, Geyer HB, Hahne FJW. Quasi-Hermitian operators in quantum mechanics and the variational principle. Ann Phys 1992;213:74-101.

[2] Bender CM, Boettcher S. Real spectra in non-Hermitian Hamiltonians having $\mathcal{P} \mathcal{T}$ symmetry. J Math Phys 1998;80(24):5243-5246.

[3] Bender CM, Boettcher S, Meisinger PN. $\mathcal{P} \mathcal{T}$-symmetric quantum mechanics. J Math Phys 1999;40(5):2201-2229.

[4] Proceedings edited by M Znojil. 1st International Workshop on Pseudo-Hermitian Hamiltonians in Quantum Physics. In: Czech. J. Phys.. vol. 54; 2004. p. 1-156.

[5] Proceedings edited by M Znojil. 2nd International Workshop on PseudoHermitian Hamiltonians in Quantum Physics. In: Czech. J. Phys.. vol. 54; 2004. p. $1005-1148$. 
[6] Proceedings edited by M Znojil. 3rd International Workshop on Pseudo-Hermitian Hamiltonians in Quantum Physics. In: Czech. J. Phys.. vol. 55; 2005. p. 10451192.

[7] Bender CM, Brody DC, Jones HF. Complex extension of quantum mechanics. Phys Rev Lett 2002;89:270401.

[8] Mostafazadeh A. Pseudo-Hermiticity versus PT symmetry: The necessary condition for the reality of the spectrum of a non-Hermitian Hamiltonian. J Math Phys 2002;43:205-214.

[9] Mostafazadeh A. Pseudo-Hermiticity versus PT symmetry: II. A complete characterization of non-Hermitian Hamiltonians with a real spectrum. J Math Phys 2002;43:2814-2816.

[10] Mostafazadeh A. Pseudo-Hermiticity versus PT symmetry: III. Equivalence of pseudo-Hermiticity and the presence of antilinear symmetries. J Math Phys 2002;43:3944-3951.

[11] Mostafazadeh A. Hilbert space structures on the solution space of Klein-Gordontype evolution equations. Class Quantum Grav 2003;20:155-171.

[12] Bender CM, Meisinger PN, Wang Q. Calculation of the hidden symmetry operator in $\mathcal{P} \mathcal{T}$-symmetric quantum mechanics. J Phys A 2003;36:1973-1983.

[13] Bender CM, Brod J, Refig A, Reuter ME. The $\mathcal{C}$ operator in $\mathcal{P} \mathcal{T}$-symmetric quantum theories. J Phys A 2004;37:10139-10165.

[14] Bender CM, Brody DC, Jones HF. Scalar quantum field theory with a complex cubic interaction. Phys Rev Lett 2004;93:251601.

[15] Bender CM, Brody DC, Jones HF. Extension of $\mathcal{P} \mathcal{T}$-symmetric quantum mechanics to quantum field theory with cubic interaction. Phys Rev D 2004;70:025001.

[16] Bender CM, Brody DC, Jones HF. Erratum: Extension of $\mathcal{P} \mathcal{T}$-symmetric quantum mechanics to quantum field theory with cubic interaction. Phys Rev D 2005;71:049901(E).

[17] Mostafazadeh A, Batal A. Physical aspects of pseudo-Hermitian and $\mathcal{P} \mathcal{T}$ symmetric quantum mechanics. J Phys A 2004;37:11645-11679.

[18] Bender CM, Cavero-Pelaez I, Milton KA, Shajesh KV. $\mathcal{P} \mathcal{T}$-symmetric quantum electrodynamics. Phys Lett B 2005;613:97-104.

[19] Mostafazadeh A. $\mathcal{P} \mathcal{T}$-symmetric cubic anharmonic oscillator as a physical model. J Phys A 2005;38:6557-6569.

[20] Kretschmer R, Szymanowski L. Quasi-Hermiticity in infinite-dimensional Hilbert spaces. Phys Lett A 2004;325:112-117.

[21] Albeverio S, Fei SM, Kurasov P. Point interactions: $\mathcal{P} \mathcal{T}$-Hermiticity and reality of the spectrum. Lett Math Phys 2002;59:227-242.

[22] Fei SM. Exactly solvable many-body systems and pseudo-Hermitian point interactions. Czech J Phys 2004;54:43-49.

[23] Znojil M, Jakubský V. Solvability and $\mathcal{P} \mathcal{T}$-symmetry in a double-well model with point interactions. J Phys A 2005;38:5041-5056.

[24] Adams RA. Sobolev Spaces. New York: Academic Press; 1975.

[25] Kato T. Perturbation Theory for Linear Operators. Berlin: Springer-Verlag; 1966. 
[26] Znojil M. $\mathcal{P} \mathcal{T}$-symmetric square well. Phys Lett A 2001;285:7-10.

[27] Znojil M, Lévai G. Spontaneous breakdown of $\mathcal{P} \mathcal{T}$-symmetry in the solvable square-well model. Mod Phys Lett A 2001;16:2273-2280.

[28] Dorey P, Dunning C, Tateo R. Spectral equivalences, Bethe ansatz equations, and reality properties in $\mathcal{P} \mathcal{T}$-symmetric quantum mechanics. J Phys A 2001;34:56795704.

[29] Langer H, Tretter Ch. A Krein space approach to PT-symmetry. Czech J Phys 2004;54:1113-1120.

[30] Caliceti E, Graffi S, Sjöstrand J. Spectra of PT-symmetric operators and perturbation theory. J Phys A 2005;38:185-193.

[31] Shin KC. On the reality of the eigenvalues for a class of $\mathcal{P} \mathcal{T}$-symmetric oscillators. Commun Math Phys 2002;229:543-564. 